\title{
Fasciolosis aguda: presentación de un caso de Cuba
}

\author{
Carmen Escalona, José Alfonso de León, María Álvarez y Roberto Cañete
}

\section{Acute fasciolosis: a Cuban case report}

Fasciolosis is a worldwide parasitic disease in human and domestic animals. The causative agents of fasciolosis are Fasciola hepatica and Fasciola gigantica but, in Cuba, only F. hepatica has been notified. As others neglected diseases, $F$. hepatica is more common in people living in rural areas where infected animals, intermediate hosts and susceptible hosts coexist. We describe the clinical features of a case that could contribute to the process of continuing medical education of health professionals in our geographic area.

Key words: Fasciola hepatica, parasitic infection.

Palabras clave: Fasciola hepatica, infección parasitaria.

\section{Introducción}

$\mathrm{F}$ asciolosis es la infección accidental de los humanos por Fasciola hepatica (F. hepatica) y Fasciola gigantica. F. hepatica fue el primer trematodo parásito conocido (Linnaeus-1758) y puede infectar humanos en todos los continentes (excepto la antártica). En contraste, $F$. gigantica se muestra más localizada en África, Medio Oriente y Asia ${ }^{1}$. F. hepatica es considerada entre las enfermedades mas desatendidas, pese a que aproximadamente 91,1 millones de humanos habitan en zonas de riesgo y de ellos 17 millones se encuentran infectados en la actualidad ${ }^{1}$. Conocida también como distomatosis hepática, la entidad zoonótica es común en los animales herbívoros y mucho menos frecuente en el humano ${ }^{1,2}$.

En Cuba se han notificado históricamente brotes de $F$. hepatica y el país cuenta con un sistema de vigilancia y atención que permite el correcto manejo de la parasitosis en todos los niveles de atención del sector salud. El primer caso de infección humana fue notificado en Cuba en 1931 y para 1944, más de 100 nuevos casos habían sido identificados lo que representaba alrededor del 33\% de las infecciones reconocidas para entonces a escala global $^{3}$. En la actualidad, casos esporádicos de fasciolosis por $F$. hepatica continúan siendo diagnosticados, particularmente en la región central y occidental del país. La incidencia anual es lo suficientemente baja como para que la parasitosis no sea considerada como un problema de salud en la isla ${ }^{3}$.

Para que ocurra la transmisión de esta parasitosis es necesaria la presencia en tiempo y espacio de los animales infectados, los moluscos dulceacuícolas que actúan como hospederos intermediarios y el hospedero susceptible. Galba cubensis y Pseudosuccinea columella, dos especies de la familia Lymnaeidae, son los hospederos interme- diarios de esta parasitosis en $\mathrm{Cuba}^{4-6}$. El ciclo de vida del parásito es complejo. Los huevos de F. hepatica son depositados inmaduros en las vías biliares desde donde son arrastrados al intestino y de ahí llevados al exterior con las heces del hospedero definitivo. Para que embrionen es imprescindible el contacto de los huevos con agua dulce a temperaturas que oscilen entre $\operatorname{los} 22-26^{\circ} \mathrm{C}$. Luego de una a dos semanas el huevo se rompe y da paso a la primera forma larvaria (miracidium) que necesita, para continuar su ciclo natural, la presencia de moluscos dulceacuícolas que actúan como hospederos intermediarios ${ }^{5}$.

En los hospederos intermediarios se producen estadios larvarios sucesivos que finalmente culminan con la liberación de las cercarías que nadan libremente hasta alcanzar plantas acuáticas, cortezas de árboles y otras superficies lisas o quedar suspendidas en aguas poco profundas donde pierden su cola y se transforman en metacercarias (blanquecinas, redondeadas y cubiertas de pared gruesa) que constituyen la forma infectante de la parasitosis ${ }^{1}$.

Cuando los mamíferos, entre ellos los humanos, consumen plantas contaminadas (fundamentalmente berros) y/o aguas infectadas con metacercarias, esos estadios larvarios llegan al duodeno y liberan el pequeño dístoma que atraviesa la pared intestinal, el peritoneo y la capsula de Glisson para más tarde llegar al parénquima hepático y luego a las vías biliares donde quedan definitivamente y alcanzan la madurez en un período aproximado de tres meses. Luego de madurar se inicia la producción de huevos y se inicia el ciclo.

Las manifestaciones clínicas asociadas con $F$. hepatica están estrechamente relacionadas con la fase en que se encuentre la infección ${ }^{7}$. Dependiendo de la carga parasitaria, la mayoría de los casos cursan sin síntomas y/o signos que evidencien la presencia del agresor, por lo que la información que pudieran aportar los antecedentes epidemiológicos y los medios diagnósticos serían
Hospital Comandante Faustino Pérez. Matanzas, Cuba (CE, JAL, MA)

Centro Provincial de Higiene, Epidemiología y Microbiología Matanzas e Instituto Cubano de Gastroenterología. La Habana. Cuba. (RC).

No existe conflicto de intereses.

Los gastos relacionados con la atención del paciente y los salarios del personal de asistencia fueron asumidos por el Hospital Comandante Faustino Pérez y el Centro Provincial de Higiene, Epidemiología y Microbiología. Matanzas, Cuba.

Recibido: 6 de diciembre de 2011 Aceptado: 9 de julio de 2012

Correspondencia a: Roberto Cañete roberto.villafranca@infomed.sld.cu 
complementos importantes a la hora de identificar a los posibles infectados ${ }^{8,9}$.

Considerando la existencia de brotes esporádicos de esa parasitosis en Cuba, así como la existencia de condiciones favorecedoras para la transmisión de $F$. hepatica en diferentes localidades rurales del país, se realiza la presentación de este caso. Con ella, se pretende colaborar con los programas de educación médica continuada que se ejecutan en Cuba y en nuestra área geográfica, e incentivar el debate en torno a esta enfermedad desatendida.

\section{Caso clínico}

Motivo de ingreso: dolor abdominal.

Centro de asistencia: Hospital Comandante Faustino Pérez. Matanzas, Cuba.

Historia de la enfermedad actual: Paciente DAV, sexo femenino, raza negra y 35 años de edad, procedente del municipio Pedro Betancourt en la provincia de MatanzasCuba. Refiere consumir con regularidad berro, lechugas y otros productos vegetales silvestres. Ingiere agua sin hervir o purificar.

A inicios de abril de 2011 presentó dolor intenso y constante en todo el abdomen, que luego de una semana se centró en epigastrio e hipocondrio derecho. El dolor se acompañó siempre de nauseas, vómitos y compromiso del estado general. Durante ese período se constató la presencia de un cuadro diarreico de tipo alto (escasas en número, abundantes en cantidad, que flotaban en el agua del inodoro y con apariencia brillosa) de aproximadamente una semana de duración, que se resolvió sin intervención medicamentosa.

Desde el inicio del proceso, se constató fiebre de 37 a 38 grados centígrados que se extendió por un período de 8 semanas, hasta mediados de mayo. Al momento del ingreso, se observó la presencia de palidez cutáneo-mucosa, abdomen globuloso y doloroso a la palpación superficial y profunda, más intenso en hipocondrio derecho y epigastrio. A los 33 días del ingreso, se detecta hepatomegalia de aproximadamente $8 \mathrm{~cm}$, con superficie lisa pero muy dolorosa, sin esplenomegalia.

El leucograma mostró un conteo global de 30.000/ $\mathrm{mm}^{3}$ con eosinofilia $81 \%$, hemoglobina $10,0 \mathrm{~g} / \mathrm{d}$ y hematocrito $0,33 \mathrm{~g} / \mathrm{l}$. Lamina Periférica: plaquetas adecuadas, leucocitosis moderada, eosinofilia marcada, hipocromía, normocitosis. TGP: 85,5 u/l, TGO: 43 u/l, FA: 348, GGT: $80 \mathrm{u} / \mathrm{l}$, amilasa sérica $63,3, \mathrm{LDH} ; 434 \mathrm{u} / \mathrm{L}$. Se realizaron 3 hemocultivos que resultaron todos negativos.

En varios exámenes de materia fecal (realizados todos en diferentes momentos, durante abril de 2011 en el laboratorio provincial de referencia en Parasitología, del Centro Provincial de Higiene, Epidemiologia y Microbiología-Matanzas) utilizando la técnica de examen directo de heces con lugol, eosina y solución salina, así como las técnicas de concentración de Willis- Malloy, Ritchie y Kato-Katz, no se observó presencia de parásitos intestinales. Se realizaron diferentes exámenes utilizando la técnica de concentración por sedimentación simple de copa- cónica que también fueron negativos.

El examen ultrasonografico demostró la existencia de hepatomegalia difusa a predominio del lóbulo izquierdo, que sobrepasaba la línea mamaria de ese lado. Igualmente se refirió que su textura se encontraba alterada con aspecto difuso y aumento de la ecogenicidad en los vasos venosos y aumento del tejido conectivo. La vesícula biliar presentaba paredes finas y no se demostró dilatación de las vías intra o extra hepáticas.

Tomografía axial computarizada: Hígado aumentado de tamaño, de aspecto heterogéneo con múltiples imágenes hipo densas de contornos irregulares distribuidos por todo el parénquima. Vesícula alitiásica de paredes finas. Bazo, páncreas y riñones sin alteraciones. En base pulmonar Izquierda, se observa pequeño derrame pleural de forma laminar.

Examen laparoscópico: aumento de tamaño del hígado que se muestra de color rojo pardo con superficie irregular y con lesiones redondeadas blanco amarillentas, rodeadas por una zona de enrojecimiento en la superficie de ambas caras de los dos lóbulos. Se demuestra la existencia de borde romo con consistencia aumentada. Bazo tamaño normal. Vesícula biliar sub- hepática blanquecina y vacía.

La detección de antígenos de excreción- secreción de $F$. hepatica (ELISA) realizado la primera semana del mes de junio de 2011 en el laboratorio de referencia del Instituto de Medicina Tropical Pedro Kouri en La Habana- Cuba, resultó positivo en heces pero no en sangre. La detección de anticuerpos IgG específicos contra el parasito resultó positiva.

Con la información clínica y epidemiológica recibida y considerando los resultados aportados por los diferentes medios diagnósticos, el equipo médico responsable decidió tratar a la paciente como un caso de fasciolosis y administró en dosis única de $20 \mathrm{mg} / \mathrm{kg}$ por vía oral luego de la ingestión de una comida rica en grasas.

Al tercer día luego de recibir el tratamiento se decidió firmar el alta y referir a la paciente a su área de salud donde se mantuvo bajo observación médica por su médico general.

Cada dos meses se realizaron exámenes de hemograma, transaminasas hepáticas y amilasemia, parámetros que regresaron a la normalidad al cuarto mes. De igual forma, se realizaron exámenes de heces mediante Copa cónica en el laboratorio provincial de referencia con resultados siempre negativos. La detección de anticuerpos IgG específicos contra el parasito mostró una tendencia descendente al ser repetido a los dos y seis meses posteriores al tratamiento con triclabendazol y la detección de 
antígenos de excreción- secreción de $F$. hepatica realizado a los dos meses de tratamiento resultó negativa en las heces y en sangre.

Seis meses luego del tratamiento la paciente no presentaba síntomas y/o signos que sugirieran la presencia del parásito. Actualmente, goza de excelente salud y se ha reinsertado a sus actividades cotidianas.

\section{Discusión}

Las enfermedades tropicales desatendidas u olvidadas constituyen un grupo de enfermedades infecciosas que se presentan con mayor frecuencia en regiones donde predomina la pobreza y la marginalidad, especialmente en población rural o en aquella que, aún radicando en zonas consideradas como urbanas, son menos favorecidas por las políticas sociales ${ }^{10}$. Esas enfermedades persisten como consecuencia de condicionantes que permiten, tanto al hospedero intermediario como al parásito, tomar ventaja en su relación con los hospederos definitivos. Es así como la pobreza a escala comunitaria o nacional y las desigualdades imperantes en muchas regiones del planeta contribuyen a la perpetuación y reemergencia de enfermedades como la fasciolosis ${ }^{11}$.

La importancia de $F$. hepatica en el desarrollo de la Parasitología es enorme. Fue el primer tremátodo descrito, cuyo ciclo se completó experimentalmente, lo que permitió y estimuló la investigación de otros parásitos en el hombre. En América, esta parasitosis cobra mayor importancia al afectar a los humanos por diferentes vías: la salud individual y colectiva, al tiempo que afecta también el desarrollo económico y la alimentación, debido a la disminución en la capacidad de producción de leche de los animales infectados, decomisos de vísceras, etc ${ }^{12}$.

Casos esporádicos de fasciolosis por $F$. hepatica han sido diagnosticados en Cuba; particularmente en la región central y occidental del país, sin embargo, la incidencia anual es lo suficientemente baja como para que la parasitosis no sea considerada un problema de salud en la isla ${ }^{3}$. No obstante, los esfuerzos realizados por la salud pública Cubana y sus logros reconocidos a escala internacional, así como el desarrollo social alcanzado, no quedan dudas acerca de la existencia de condiciones que permiten, aún en la actualidad, la trasmisión de diferentes parasitosis y sus efectos deletéreos hacia la salud de los afectados ${ }^{13}$. La mayor prevalencia de $F$. hepatica a escala global, se notifica en Bolivia, Ecuador, Perú, Portugal y España, aunque es también elevada en Turquía, Egipto, algunas regiones de África, Irán y Vietnam ${ }^{1,14}$.

El caso que se presenta, corresponde a una mujer del municipio Pedro Betancourt, en el que es frecuente la ingestión a escala comunitaria de berro, lechugas y otros productos vegetales de origen silvestre. Es tan común encontrar berro en ese sitio, que los habitantes no tienen necesidad de invertir recursos económicos en adquirirlo debido a que crece indiscriminadamente en cualquier alcantarillado, zanja e incluso en los sitios donde se producen escapes de agua, como por ejemplo: tanques elevados o en la periferia de huertos escolares. Es común también en ese municipio (clasificado como rural), la coexistencia espacial de las personas con ganado vacuno o caprino y con moluscos dulceacuícolas (Galba cubensis y Pseudosuccinea columella), hospederos intermediarios de esta parasitosis.

La progresión de los síntomas y signos clínicos en la paciente, nos hace pensar que transitó de la fase aguda a la crónica en el período de su hospitalización. La aseveración es consecuente con los exámenes de heces negativos, utilizando diferentes técnicas diagnósticas, durante el mes de abril y la positividad de esos mismos exámenes y de los antígenos de excreción-secreción del parásito en heces en el mes de junio. Los antígenos son positivos en sangre durante la fase aguda de la infección y solo se hacen positivos en heces, cuando se ha pasado a la fase crónica, etapa en que se negativizan en la sangre, como sucedió en la paciente descrita. La determinación de anticuerpos específicos contra la parasitosis, será positiva a las 2 ó 3 semanas posteriores a la ingestión de los elementos parasitarios (metacercarias en vegetales acuáticos) y por ello, resultó positiva en este caso.

Debido a que el dolor abdominal y la eosinofilia son comúnmente identificados en individuos infectados por $F$. hepatica el diagnóstico diferencial debe hacerse con otras parasitosis como ascariosis, ancilostomosis y estrongiloidosis. También deben ser considerados los abscesos hepáticos, las hepatitis víricas y el cuadro desencadenado por la larva migrans visceral ${ }^{9,10}$.

Como toda enfermedad de transmisión digestiva la educación sanitaria de la comunidad, el tratamiento efectivo de los individuos ya afectados y el control higiénico del medio ambiente serian las piedras angulares para prevenir la aparición de nuevos casos.

Dentro de las acciones que deben ejecutarse se podrían mencionar: a) la educación de la población de las zonas de riesgo para que no coma berros u otras plantas acuáticas silvestres o de origen desconocido a no ser que sean adecuadamente cocidos, en especial, en zonas donde la enfermedad es altamente prevalente en ganado; b) no utilizar excremento de ganado sin tratamiento previo para fertilizar las plantas acuáticas que posteriormente serán destinadas para consumo humano; c) drenar la tierra o utilizar molusquicidas químicos para eliminar los hospederos intermediarios de F. hepática y d) tratamiento antihelmíntico al ganado infectado.

La preparación y pericia clínica de los profesionales de la salud, unida a la información epidemiológica y a los aportes de los diferentes medios diagnósticos, son 
los elementos cardinales de una buena atención médica. En este caso, las acciones de las diferentes secciones del hospital de asistencia y su integración con otras unidades del sector salud en Cuba (Centro Provincial de Higiene, Epidemiologia y Microbiología- Matanzas y el Instituto de Medicina Tropical Pedro Kouri-La Habana), permitieron la identificación del agente causal de la entidad y luego, el adecuado tratamiento medicamentosos con triclabendazol, así como su seguimiento en el área de salud por el médico de la comunidad, los miembros del departamento de educación y educación para la salud y el epidemiólogo del área.

Era evidente que se necesitaba un programa de intervención comunitaria para prevenir la aparición de nuevos casos y realizar una pesquisa activa para diagnosticar aquellos que aun teniendo la infección, no habían sido identificados por los profesionales de la salud. El Centro Provincial de Higiene, Epidemiología y Microbiología de la provincia de Matanzas activó sus diferentes secciones y durante 3 meses encaminó sus esfuerzos en una campaña de información local, que permitió recibir en una consulta especializada creada para ese fin, a otros casos y ofrecerles el tratamiento con triclabendazol y la información pertinente para evitar la reinfección.

Se orientó a las autoridades locales, para la implementación de acciones dirigidas al tratamiento de los animales infectados y la atención a los sitios identificados como positivos a los moluscos dulceacuícolas, hospederos intermediarios de F. hepatica.

El diagnóstico y tratamiento oportuno, constituyen elementos cardinales en el manejo de las infecciones por $F$. hepatica ${ }^{15}$. Con ello no solo se evitarían lesiones hepáticas irreversibles en individuos aislados, sino que se evitaría la progresión de brotes que reducirían la calidad de vida en las comunidades afectadas y se disminuirían los costos de la atención sanitaria. El conocimento de las parasitosis que potencialmente afectan a las comunidades, así como la integración de los elementos epidemiológicos, con la información que ofrecen los diferentes medios diagnósticos, garantizará la atención oportuna y eficiente de las necesidades sanitarias de la población.

\section{Resumen}

Fasciolosis es una enfermedad parasitaria de humanos y diferentes animales. Sus agentes causales son Fasciola hepatica y Fasciola gigantica; sin embargo, en Cuba, solo la primera de esas especies ha sido notificada. Como otras enfermedades incorporadas en el grupo de las olvidadas o desatendidas, fasciolosis, es más frecuente en personas que viven en áreas rurales donde coexisten animales infectados, hospederos intermediarios y hospederos susceptibles. Se exponen las características de un caso que pudiera contribuir en el proceso de educación continuada de los profesionales de la salud en nuestra área geográfica.

\section{Referencias bibliográficas}

1.- Tolan RW. Fascioliasis Due to Fasciola hepatica and Fasciola gigantica Infection: An Update on This 'Neglected' Neglected Tropical Disease. Laboratory Medicine 2011; 42 (2): 107- 16.

2.- World Health Organization. The "Neglected" Neglected Worms. Action Against Worms. December 2007, issue 10. Available at: www. who.int/neglected_diseases/preventive_ chemotherapy/Newsletter10.pdf. Accessed July 6, 2010.

3.- Rojas L, Vázquez A, Domenech I, Robertson L J. Fascioliasis: can Cuba conquer this emerging parasitosis? Trends Parasitol 2010; 26 (1): 26-34.

4.- Cañete R, Gutiérrez A, Sánchez J. Distribución y diversidad de moluscos fluviales en un área rural con incidencia de Fasciolosis del municipio San Juan y Martínez, Pinar del Rio, Cuba. J Med Appl Malacol 2001-2002; 11: 1-6.

5.- Cañete R, Yong M, Sánchez J, Wong L,
Gutiérrez A. Population Dynamics of Intermediate Snail Hosts of Fasciola hepatica and Some Environmental Factors in San Juan y Martinez Municipality, Cuba. Mem Inst Oswaldo Cruz 2004; 99 (3): 257-62.

6.- Gutiérrez A, Vázquez A A, Hevia Y, Sánchez J, Correa A C, Hurtrez-Boussès S, et al. First report of larval stages of Fasciola hepatica in a wild population of Pseudosuccinea columella from Cuba and the Caribbean. J Helminthol 2011; 85 (1): 109-11.

7.- Mas-Coma S. Epidemiology of fascioliasis in human endemic areas. J Helminth 2005; 79 (3): 207- 16.

8.- Keiser J, Utzinger J. Food-borne trematodiases. Clin Microbiol Rev 2009; 22: 466-83.

9.- Keiser J, Duthaler U, Utzinger J. Update on the diagnosis and treatment of food-borne trematode infections. Curr Opin Infect Dis 2010; 23 (5): 513-20.

10.- Hotez P J, Kamath A. Neglected Tropical Diseases in Sub-Saharan Africa: Review of Their Prevalence, Distribution, and Disease
Burden. PLoS Negl Trop Dis 2009; 3 (8): e412. doi:10.1371/journal.pntd.0000412.

11.- Manderson L, Aagaard-Hansen J, Allotey P, Gyapong M, Sommerfeld J. Social Research on Neglected Diseases of Poverty: Continuing and Emerging Themes. PLoS Neg1 Trop Dis 2009; 3(2):e332. doi:10.1371/journal. pntd.0000332.

12.- Espinoza J R, Terashima A, Herrera-Velit P, Marcos L A. Human and animal fascioliasis in Peru: impact in the economy of endemic zones. Rev Peru Med Exp Salud Publica 2010; 27 (4): 604-12.

13.- Escobedo A A, Cañete R, Núñez F A. Intestinal protozoan and helminth infections in the Municipality San Juan y Martínez, Pinar del Río, Cuba. Trop Doct 2007; 37 (4): 236-8.

14.- Sezgın O, Altintaş E, Tombak A, Uçbılek E. Fasciola hepatica-induced acute pancreatitis: report of two cases and review of the literature. Turk J Gastroenterol 2010; 21 (2): 183-7.

15.- Cañete R, Noda A L, Domenech I, Brito K. Infección por Fasciola hepatica y fasciolosis. Rev Panam Infectol 2011; 13 (4): 33-9. 